\title{
(一)-牛蒡苷元及其对映异构体的不对称合成新方法
}

\author{
吴平徐凯付莹康廷国窦德强翟延君* \\ (辽宁中医药大学药学院 大连 116600)
}

\begin{abstract}
摘要 (一)-牛蒡苷元属于二芐基丁内酯型木脂素, 是中药牛蒡子的主要活性成分. 为了研究牛蒡苷元的构效关系, 报 道了(一)-牛蒡苷元及其对映异构体的不对称合成新方法. 以苯丙酸衍生物为起始原料, 首先利用噁唑烷酮类手性辅基 构建丁内酯 $\beta$ 位的手性中心, $R$ 构型和 $S$ 构型 $\beta$-苄基丁内酯的 $e e$ 值分别为 $98 \%$ 和 $96 \%$. 再利用空间位阻效应在 $\alpha$ 位构 建第二个手性中心，最后脱除保护基得到目标产物. 经 6 步反应，分别以 58\%、55\%的总收率和 97\%、96\%的 $e e$ 值得到 (一)-牛蒡苷元和(十)-牛蒡苷元. 为接下来拟进行的结构优化奠定了技术基础.
\end{abstract}

关键词 牛蒡苷元; 噁唑烷酮; Evans; 木脂素; 丁内酯; 不对称合成

\section{A New Method for Asymmetric Synthesis of (-)-Arctigenin and Its Enantiomer}

\author{
Wu, Ping Xu, Kai Fu, Ying Kang, Tingguo Dou, Deqiang Zhai, Yanjun* \\ (School of Pharmacy, Liaoning University of Traditional Chinese Medicine, Dalian 116600)
}

\begin{abstract}
Arctigenin, the main active ingredient of traditional chinese medicine (TCM) arctii fructus, belongs to dibenzyl butyrolactone lignans. In order to study the structure-activity relationship of arctigenin, a new method for asymmetric synthesis of (-)-arctigenin and its enantiomer was developed. Phenylpropanoic acid derivate was used as starting material and the chiral center of beta butyrolactone was constructed by using oxazolidinone chiral auxiliary. The ees of $R$ and $S$ configurations are $98 \%$ and $96 \%$, respectively. Then the second chiral center in the alpha position was constructed benefitting from the steric effect. After removal of protecting group, the target compounds were obtained in $58 \%$ and $55 \%$ overall yield of $(-)$-arctigenin and (+)-arctigenin with $97 \%$ and $96 \%$ ee, respectively. This work paved the way for further structural optimization of arctigenin.
\end{abstract}

Keywords arctigenin; oxazolidinone; Evans; lignan; butyrolactone; asymmetric synthesis

(一)-牛蒡苷元 (1)，(3R,4R)-3-(3-甲氧基-4-羟基苄 基)-4-(3,4-二甲氧基苠基)-丁内酯, 是中药牛蒡子(Arctii Fructus)的最主要活性成分(图 1). 研究表明, (一)-牛蒡 甘元具有抗炎 ${ }^{[1]}$ 、抗心律失常 ${ }^{[2] 、}$ 降血糖 ${ }^{[3]}$ 、保护神经 ${ }^{[4]}$ 、 抗肿瘤 ${ }^{[5]}$ 、缓解内质网应激 ${ }^{[6]}$ 、抗老年痴呆 ${ }^{[7]}$ 等多种生物 活性, 因此近年来受到了国内外越来越多的科研工作者 的关注 ${ }^{[8]}$. 本课题组长期致力于中药牛蒡子的开发研究, 包括牛蒡子主要活性成分(一)-牛蒡苷元的衍生化 ${ }^{[9]}$. 本 课题组最近在先前发现牛蒡苷元具有较好神经保护作 用的基础上又研究了其可能的作用机理 ${ }^{[10]}$. 为了系统 深入地研究牛蒡苷元的构效关系, 利用化学方法对其进
行不对称全合成具有重要的意义. 通过综合分析现有

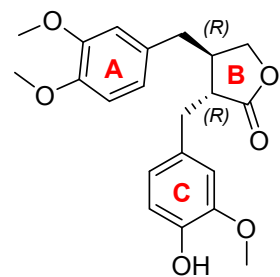

(-)-Arctigenin (1)<smiles>COc1cc(C[C@H]2C(=O)OCC2Cc2ccc(OC)c(OC)c2)ccc1O</smiles>

(+)-Arctigenin (2)
图 1 (一)-牛蒡苷元及其对映异构体结构

Figure 1 Structure of $(-)$-arctigenin and its enantiomer

*E-mail: wupingtcm@163.com

Received November 19, 2015; revised December 21, 2015; published online January 15, 2016.

Project supported by the National Natural Science Foundation of China (No. 30873437) and the Ph.D. Program Foundation of the Ministry of Education of China (No. 20112133110001)

国家自然科学基金(No. 30873437)和国家教育部博士点基金(No. 20112133110001)资助项目. 
(一)-牛蒡苷元的不对称合成路线 ${ }^{[11]}$, 我们决定开发一条 更适于大量制备及衍生物快速合成的新路线.

\section{1 结果与讨论}

(一)-牛蒡苷元不对称合成新方法如 Scheme 1 所示. 以 3,4-二甲氧基苯丙酸(3)为起始原料, 与手性辅基 4-芐 基噁唑烷酮 4 缩合得化合物 $\mathbf{5}$, 随后在大位阻有机碱双 (三甲基硅基)氨基钠(NaHMDS)的作用下发生 Evans 辅 基介导的羰基 $\alpha$-烷基化反应, 得到化合物 $\mathbf{6}$, 还原消除 手性辅基后得到醇 7. 在对甲苯磺酸催化下发生酯交换 反应得到 $e e$ 值分别为 $98 \%$ 的丁内酯 $\mathbf{8 a}$ (文献报道 ${ }^{[11]}$ 利用 其它路线合成 8a, $e e$ 值为 $91 \% \sim 96 \%$ 不等)和 $96 \%$ 的 $8 \mathbf{b}$, 随后再利用大位阻有机碱二异丙基胺基锂(LDA)在 $\mathbf{8}$ 的 酯羰基 $\alpha$ 位引入衍生化的茮基得到 10a/10b ( $e>>99 \%$ ), 最后 $\mathrm{Pd} / \mathrm{C}$ 催化氢解除去酚羟基保护基, 分别以 6 步 $58 \% 、 55 \%$ 的总收率和 $97 \% 、 96 \%$ 的 ee 值得到目标产物 $(-)$ - 牛蒡苷元 1 和 $(+)$-牛蒡苷元 2.

化合物 5 和 8 羰基 $\alpha$-烷基化立体选择性的模型如图 2 所示. 当钠离子与化合物 $\mathbf{5 a}$ 发生配位后, 由于茮基的 位阻作用, 溴乙酸叔丁酯的羰基 $\alpha$ 碳主要从 $R e$ 面进攻, 主产物为 $\mathbf{6 b}$, 而从 $S i$ 面进攻则为副产物. 根据化合物 $\mathbf{8 b}$ 的 $e e$ 值测定结果, $R e$ 面进攻产物与 $S i$ 面进攻产物的
比值为 $98 ： 2$. 同理，由于化合物 5a 中 Evans 辅基上苄 基构型, 溴乙酸叔丁酯的羰基 $\alpha$ 碳主要从 $S i$ 面进攻, 根 据 $8 \mathbf{a}$ 的 $e e$ 值测定结果, $S i$ 面进攻产物与 $R e$ 面进攻产物 的比值为 $99: 1$.

五元环内稀醇的非对映选择性烷基化反应中, anti诱导的烷基化过渡态主要受到空间位阻的控制方式所 支配 ${ }^{[12]}$. 对于化合物 8 而言, 由于丁内酯 $\beta$ 位已经有了 一个位阻较大的基团，所以同样具有大位阻作用的取代 溴苄的亚甲基碳只能从相反的方向进攻生成相应的化 合物 10, 同侧进攻则生成 $s y n$-异构体副产物. 由于 $\mathrm{Pd} / \mathrm{C}$ 氢解不涉及到手性问题, 根据化合物 $\mathbf{1} / \mathbf{2}$ 的光学纯度测 定结果, anti-诱导产物与 $s y n$-异构体的比例大于 $99: 1$.

Scheme 1 所示路线适用于牛蒡苷元 C 环具有不同 取代基的衍生物的快速制备. 随后，我们参考文献[13] 尝试开发另一条合成路线(Scheme 2), 希望通过这条路 线制备 $(+)$-牛蒡苷元的同时, 可以以 13 或其对映异构 体为中间体快速制备 $\mathrm{A}$ 环具有不同取代基的 $\beta$-芐基丁 内酯衍生物. 然而, 当我们顺利得到中间体 13 后, 发现 随后的岸基 $\alpha$-位烷基化反应并没有区域选择性, 13 的酯 羰基 $\alpha$ 位和 $\beta$ 位均发生了反应. 我们调整有机碱的种类 和原料的当量比均未能解决该问题.<smiles>COc1ccc(CCC(=O)O)cc1OC</smiles>
3<smiles>COc1ccc(C[C@@H]2COC(=O)C2)cc1C[C@@H]1COC(=O)C1</smiles><smiles>COc1ccc(CCC(=O)N2C(=O)OCC2C(=O)c2ccccc2)cc1OC</smiles>
$5 a / 5 b$<smiles>CCOc1ccc(C[C@H]2C[C@H](Cc3ccc(OC)c(OC)c3)CC2=O)cc1OC</smiles><smiles>COc1ccc(CC2CCC(=O)C2Cc2ccc(OC)c(OC)c2)cc1OC</smiles><smiles>C1=CC=C1</smiles><smiles>COc1ccc(CC(CC(=O)OC(C)(C)C)C(=O)N2C(=O)OCC2Br)cc1OC</smiles><smiles>C1CC2CCCC2C1</smiles><smiles>COc1ccc(C[C@@H](CO)CC(=O)OC(C)(C)C)cc1OC</smiles><smiles>COc1ccc(C[C@@H]([CH]CO)CC(=O)OC(C)(C)C)cc1OC</smiles><smiles>COc1ccc(C[C@@H]2COC(=O)[C@@H]2CC(C)C)cc1OC</smiles>

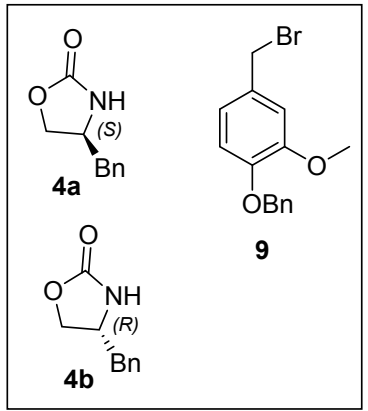

Reagents and conditions: (i) $\mathrm{PivCl}, \mathrm{Et}_{3} \mathrm{~N}, \mathbf{4 a}$ for $\mathbf{5 a}, \mathbf{4 b}$ for $\mathbf{5 b}, \mathrm{THF},-20{ }^{\circ} \mathrm{C}$ r.t., $2 \mathrm{~h}$; (ii) NaHMDS, tert-butyl bromoacetate, THF, $-78{ }^{\circ} \mathrm{C}, 2 \mathrm{~h} ; 91 \%$ for 6a, $90 \%$ for $\mathbf{6 b}$ by 2-steps; (iii) $\mathrm{NaBH}_{4}$, THF, $\mathrm{H}_{2} \mathrm{O}$, r.t., $2 \mathrm{~h}$; (iv) $p$-TsOH, toluene, $80{ }^{\circ} \mathrm{C}, 1 \mathrm{~h}$; $83 \%$ for $8 \mathbf{a}, 81 \%$ for 8 b by 2 -steps; (v) LDA, 9 , THF, $-78{ }^{\circ} \mathrm{C}$, $4 \mathrm{~h} ; \mathbf{7 9} \%$ for $\mathbf{1 0 a}, \mathbf{7 6} \%$ for $\mathbf{1 0 b}$; both $d e>99 \%$; (vi) $\mathrm{Pd} / \mathrm{C}, \mathrm{H}_{2}, \mathrm{MeOH}$, r.t., $1 \mathrm{~h}$; $98 \%$ for $\mathbf{1}, 100 \%$ for 2 .

图式 1 (一)-牛蒡苷元全合成路线

Scheme 1 Total synthetic route for $(-)$-arctigenin 

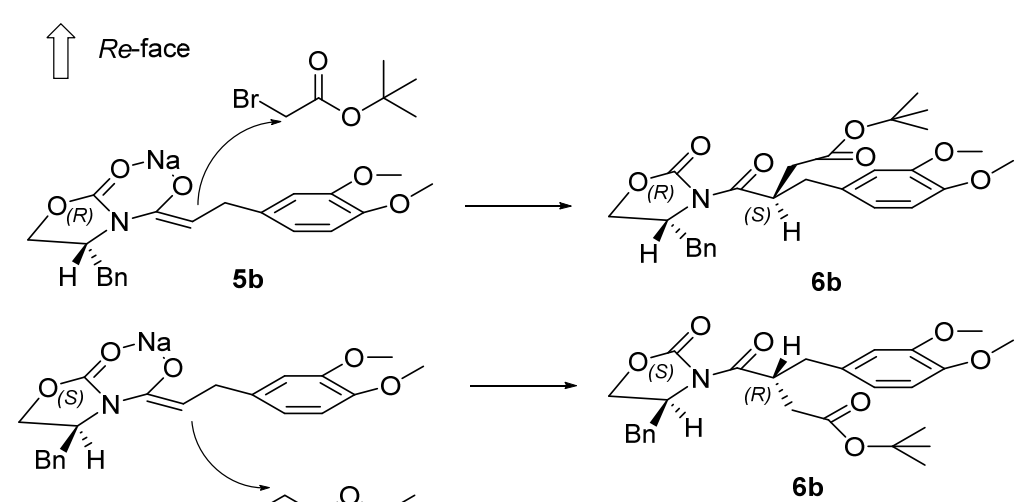

$6 b$

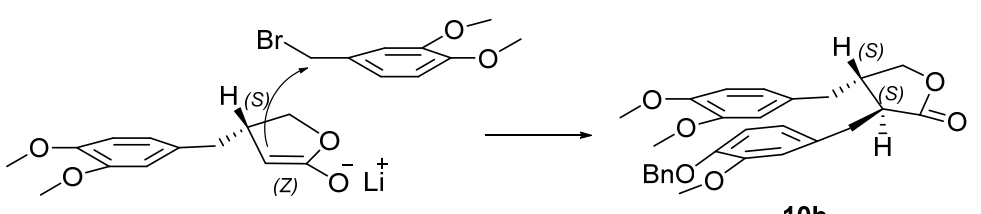

8b

$10 \mathrm{~b}$
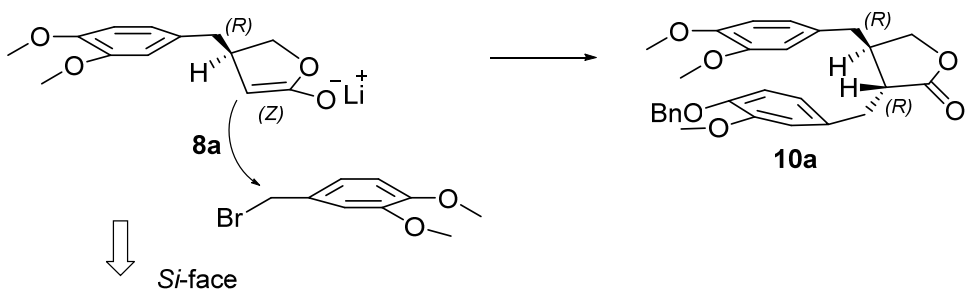

图 2 化合物 $\mathbf{5}$ 和 $\mathbf{8}$ 羰基 $\alpha$-烷基化立体选择性模型

Figure 2 Proposed models for the stereochemical course of the alkylation of $\mathbf{5}$ and $\mathbf{8}$

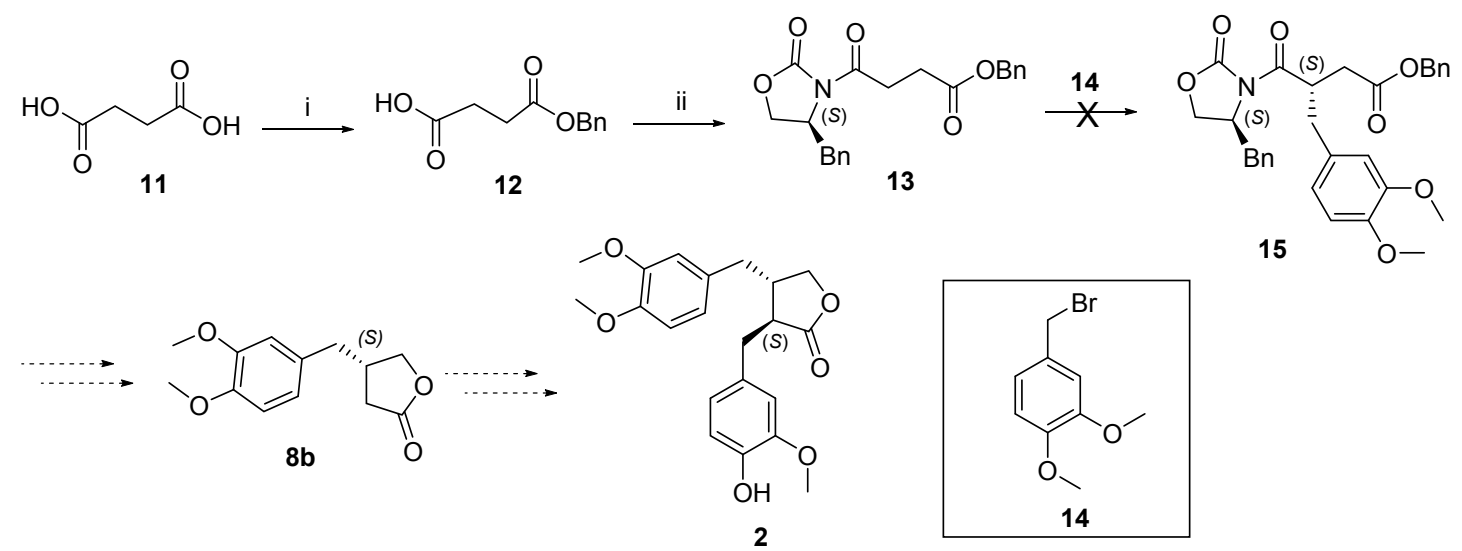

Reagents and conditions: (i) $\mathrm{BnBr}, \mathrm{Et}_{3} \mathrm{~N}$, THF, reflux, $5 \mathrm{~h}, 62 \%$; (ii) PivCl, $\mathrm{Et}_{3} \mathrm{~N}, 4 \mathrm{a}, \mathrm{THF},-20{ }^{\circ} \mathrm{C} \sim$ r.t., 2 h, $97 \%$.

图式 $2(+)-$ 牛蒡子苷元全合成路线

Scheme 2 Attempted total synthetic route for $(+)$-arctigenin

上述路线中涉及的澳茮衍生物, 按照文献[14]所述 方法制备 (Scheme 3). 以香草醛为起始原料, 经三步反 应，分别以 $82 \%$ 和 79\%的总收率得到 9 和 14.

\section{2 结论}

本文研究了用于不对称合成天然产物(一)-牛蒡苷
元及其对映异构体的新方法.

我们利用噁唑烷酮类手性辅基(Evans)成功制备了 关键中间体 $(R)$-4-(3,4-二甲氧基苄基)丁内酯 (8a) 和 $(S)-4-(3,4-$ 二甲氧基芐基)丁内酯 $(\mathbf{8 b})$, 其 $e e$ 值分别为 98\%、96\%. 并在此基础上制备了(一)-牛蒡子苷元 1 及 其对映异构体(+)-牛蒡子苷元 2 , 其 $e e$ 值分别为 $97 \%$ 、 
96\%. 为接下来拟进行的(一)-牛蒡苷元结构优化奠定了 技术基础.

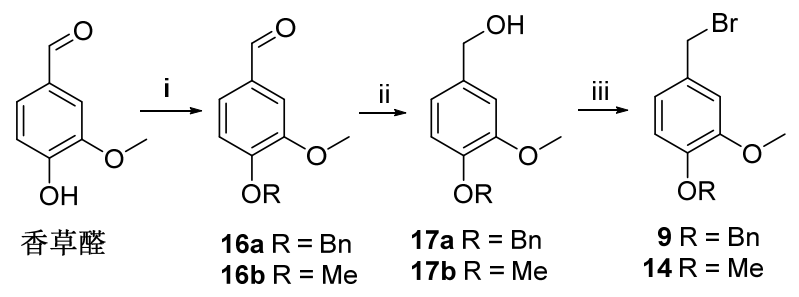

Reagents and conditions: (i) $\mathrm{K}_{2} \mathrm{CO}_{3}, \mathrm{DMF}, \mathrm{BnBr}$ for 16a and Mel for 16b, r.t., 2 h; (ii) $\mathrm{NaBH}_{4}, \mathrm{THF}, \mathrm{H}_{2} \mathrm{O}, 0{ }^{\circ} \mathrm{C}, 30$ min; (iii) $\mathrm{PBr}_{3}, \mathrm{DCM}$, $0{ }^{\circ} \mathrm{C}, 20 \mathrm{~min} ; 82 \%$ for 9 and $79 \%$ for 14 by 3 -steps.

\section{图式 3 溴苄衍生物合成路线}

Scheme 3 Synthetic route for benzyl bromide derivatives

\section{3 实验部分}

\section{1 仪器与试剂}

Bruker AM 400 型核磁共振仪(瑞士 Bruker 公司), 以 $\mathrm{CDCl}_{3}$ 为溶剂, TMS 为内标; Agilent LCMSD 质谱仪 (美国 Agilent公司); $\mathrm{AB} 5600+\mathrm{Q}$ 高分辨质谱仪(美国 $\mathrm{AB}$ SCIEX 公司); Rudolph Autopol IV-T 型自动旋光仪(美国 鲁道夫); X-4 显微熔点仪(上海精密科学仪器有限公司). 薄层层析硅胶、柱层析硅胶购自烟台江友硅胶开发有限 公司，所有试剂购自上海泰坦科技股份有限公司旗下探 索平台.

\section{2 化合物的合成}

\subsection{1 (S)-4-芳基-3-(3-(3,4-二甲氧基苯基)丙酰基)-2-} 覀唑烷酮 $(\mathbf{5 a})$ 的合成

将 3,4-二甲氧基苯丙酸 $(2.10 \mathrm{~g}, 10.0 \mathrm{mmol})$ 溶于 60 $\mathrm{mL}$ 无水 $\mathrm{THF}$ 中, 于 $-20{ }^{\circ} \mathrm{C}$ 滴加特戊酰氯 $(1.20 \mathrm{~mL}$, $10.0 \mathrm{mmol}$ )和三乙胺 $(4.20 \mathrm{~mL}, 30.0 \mathrm{mmol})$, 此温度下继 续搅拌 $20 \mathrm{~min}$. 滴加 $(S)$-4-芐基-2-噁唑烷酮(1.60 g, 9.0 $\mathrm{mmol})$ 的 THF 溶液 $(20 \mathrm{~mL})$, 一次性加入无水 $\mathrm{LiCl}(0.42$ $\mathrm{g}, 10.0 \mathrm{mmol}$ ), 继续搅拌 $20 \mathrm{~min}$ 后升至室温, 再继续摚 拌 $2 \mathrm{~h}$. 反应液减压浓缩至约 $20 \mathrm{~mL}$, 以 $100 \mathrm{~mL}$ 乙酸乙 酯稀释, 依次用 $10 \%$ 碳酸氢钠、 $5 \%$ 硫酸氢钾、饱和氯化 钠溶液洗涤, 无水硫酸钠干燥. 抽滤, 滤液减压浓缩, 得 $3.27 \mathrm{~g}$ 白色固体 $\mathbf{5 a}$, 不经纯化, 可直接用于下步反应. m.p. 88 90 ${ }^{\circ} \mathrm{C}$ (文献值 $\left.{ }^{[15]} 91.5 \sim 92{ }^{\circ} \mathrm{C}\right) ;[\alpha]_{\mathrm{D}}^{25}+45.1(c$ $\left.1.0, \mathrm{CHCl}_{3}\right) ;{ }^{1} \mathrm{H} \mathrm{NMR}\left(400 \mathrm{MHz}, \mathrm{CDCl}_{3}\right) \delta: 2.75(\mathrm{dd}, J=$ $13.3,9.6 \mathrm{~Hz}, 1 \mathrm{H}), 3.91 \sim 3.04(\mathrm{~m}, 2 \mathrm{H}), 3.16 \sim 3.35(\mathrm{~m}$, $3 \mathrm{H}), 3.86(\mathrm{~s}, 3 \mathrm{H}), 3.88(\mathrm{~s}, 3 \mathrm{H}), 4.15 \sim 4.21(\mathrm{~m}, 2 \mathrm{H}), 4.67$ (ddt, $J=9.9,6.6,3.2 \mathrm{~Hz}, 1 \mathrm{H}), 6.77 \sim 6.81(\mathrm{~m}, 3 \mathrm{H}), 7.17(\mathrm{~d}$, $J=6.7 \mathrm{~Hz}, 2 \mathrm{H}), 7.31 \sim 7.38(\mathrm{~m}, 3 \mathrm{H}) ;{ }^{13} \mathrm{C} \mathrm{NMR}(100 \mathrm{MHz}$, $\left.\mathrm{CDCl}_{3}\right) \delta: 172.6,153.6,149.0,147.6,135.3,133.2,129.6$, 129.2, 129.1, 127.5, 120.6, 112.0, 111.4, 66.3, 56.1, 56.0,
55.3, 38.0, 37.4, 30.1, 26.6; ESI-MS m/z: $370.2[\mathrm{M}+\mathrm{H}]^{+}$, $392.1[\mathrm{M}+\mathrm{Na}]^{+}$.

3.2.2 (R)-4-芐基-3-[3-(3,4-二甲氧基苯基)丙酰基]-2噯唑烷酮 $(5 b)$ 的合成

同化合物 5a 合成. 白色固体. m.p. $88 \sim 90{ }^{\circ} \mathrm{C}$; $[\alpha]_{\mathrm{D}}^{24}-45.5\left(c 1.0, \mathrm{CHCl}_{3}\right) ;{ }^{1} \mathrm{H}$ NMR $\left(400 \mathrm{MHz}, \mathrm{CDCl}_{3}\right)$ $\delta: 2.75(\mathrm{dd}, J=13.3,9.6 \mathrm{~Hz}, 1 \mathrm{H}), 2.92 \sim 3.05(\mathrm{~m}, 2 \mathrm{H})$, $3.17 \sim 3.39(\mathrm{~m}, 3 \mathrm{H}), 3.85(\mathrm{~s}, 3 \mathrm{H}), 3.88(\mathrm{~s}, 3 \mathrm{H}), 4.14 \sim 4.20$ (m, $2 \mathrm{H}), 4.61 \sim 4.75(\mathrm{~m}, 1 \mathrm{H}), 6.77 \sim 6.81(\mathrm{~m}, 3 \mathrm{H}), 7.17(\mathrm{~d}$, $J=6.7 \mathrm{~Hz}, 2 \mathrm{H}), 7.27 \sim 7.36(\mathrm{~m}, 3 \mathrm{H}) ;{ }^{13} \mathrm{C} \mathrm{NMR}(100 \mathrm{MHz}$, $\left.\mathrm{CDCl}_{3}\right) \delta: 172.6,153.6,149.0,147.6,135.3,133.2,129.5$, 129.2, 129.1, 127.5, 120.6, 112.0, 111.4, 66.3, 56.1, 56.0, 55.2, 37.9, 37.4, 30.1, 26.6; ESI-MS m/z: $370.2[\mathrm{M}+\mathrm{H}]^{+}$, $392.1[\mathrm{M}+\mathrm{Na}]^{+}$.

3.2.3 (S)-4-芐基-3-[(R)-2-乙酸叔丁酯基-3-(3,4-二甲 氧基苯基)丙酰基]-2-噯唑烷酮(6a)的合成

将上步产物 5a (2.13 g, $5.8 \mathrm{mmol}$ ) 溶于 $100 \mathrm{~mL}$ 无水 THF, 于 $-78{ }^{\circ} \mathrm{C}$ 滴加 NaHMDS $(4.3 \mathrm{~mL}, 8.6 \mathrm{mmol}, 2$ $\mathrm{mol} / \mathrm{L}$ in THF). 搅拌 $1 \mathrm{~h}$ 滴加澳乙酸叔丁酯 $(1.70 \mathrm{~mL}$, $11.5 \mathrm{mmol}$ ), 继续在此温度下搅拌 $2 \mathrm{~h}$. 饱和氯化铵溶液 淬灭反应, 减压浓缩除去大部分四氢呋喃, 剩余物溶于 $100 \mathrm{~mL}$ 乙酸乙酯, 依次用 $5 \%$ 硫酸氢钾和饱和氯化钠溶 液洗涤, 无水硫酸钠干燥. 抽滤, 滤液减压浓缩, 硅胶 柱层析 [ $V$ (石油醚) $: V$ (乙酸乙酯 $)=4: 1$ ], 得 $2.58 \mathrm{~g}$ 白色 固体 6a, 两步产率 91\%. m.p. $77 \sim 79{ }^{\circ} \mathrm{C} ;[\alpha]_{\mathrm{D}}^{25}+117.9$ (c 1.0, $\mathrm{CHCl}_{3}$ ); ${ }^{1} \mathrm{H}$ NMR (400 MHz, $\left.\mathrm{CDCl}_{3}\right) \delta: 1.40$ (s, $8.45 \mathrm{H}), 1.46$ (s, $0.55 \mathrm{H}), 2.38(\mathrm{dd}, J=16.9,3.9 \mathrm{~Hz}, 1 \mathrm{H})$, $2.56(\mathrm{dd}, J=13.1,9.4 \mathrm{~Hz}, 1 \mathrm{H}), 2.74(\mathrm{dd}, J=13.4,10.0 \mathrm{~Hz}$, $1 \mathrm{H}), 2.83$ (dd, $J=17.0,11.0 \mathrm{~Hz}, 1 \mathrm{H}), 2.96(\mathrm{dd}, J=13.1$, $6.0 \mathrm{~Hz}, 1 \mathrm{H}), 3.31(\mathrm{dd}, J=13.4,2.7 \mathrm{~Hz}, 1 \mathrm{H}), 3.85$ (s, 3H), 3.89 (s, 3H), 3.97 (t, $J=8.3 \mathrm{~Hz}, 1 \mathrm{H}), 4.10$ (dd, $J=9.0,2.2$ $\mathrm{Hz}, 1 \mathrm{H}), 4.41 \sim 4.49(\mathrm{~m}, 1 \mathrm{H}), 4.49 \sim 4.62(\mathrm{~m}, 1 \mathrm{H}), 6.77 \sim$ $6.79(\mathrm{~m}, 2 \mathrm{H}), 6.87(\mathrm{~s}, 1 \mathrm{H}), 7.26 \sim 7.29(\mathrm{~m}, 3 \mathrm{H}), 7.31 \sim$ $7.38(\mathrm{~m}, 2 \mathrm{H}) ;{ }^{13} \mathrm{C}$ NMR $\left(100 \mathrm{MHz}, \mathrm{CDCl}_{3}\right) \delta: 175.6$, $171.4,153.2,149.0,147.9,135.8,130.7,129.6,129.0$, $127.4,121.4,112.3,111.1,81.0,66.0,56.03,55.99,55.7$, 41.5, 38.1, 37.7, 36.8, 28.2; ESI-MS $m / z$ : $506.1[\mathrm{M}+\mathrm{Na}]^{+}$; HRMS (ESI-TOF) calcd for $\mathrm{C}_{27} \mathrm{H}_{33} \mathrm{NNaO}_{7}[\mathrm{M}+\mathrm{Na}]^{+}$ 506.2149, found 506.2156.

3.2.4 (R)-4-芐基-3-[(S)-2-乙酸叔丁酯基-3-(3,4-二甲 氧基苯基)丙酰基]-2-噁唑烷酮(6b)的合成

同化合物 6a 合成. 白色固体, 两步产率 $90 \%$. m.p. $77 \sim 78{ }^{\circ} \mathrm{C} ;[\alpha]_{\mathrm{D}}^{25}-117.4\left(c 1.0, \mathrm{CHCl}_{3}\right) ;{ }^{1} \mathrm{H}$ NMR $(400$ $\left.\mathrm{MHz}, \mathrm{CDCl}_{3}\right) \delta: 1.41(\mathrm{~s}, 8.7 \mathrm{H}), 1.46(\mathrm{~s}, 0.3 \mathrm{H}), 2.38(\mathrm{dd}$, $J=16.9,3.0 \mathrm{~Hz}, 1 \mathrm{H}), 2.56(\mathrm{dd}, J=12.5,9.7 \mathrm{~Hz}, 1 \mathrm{H}), 2.75$ 
(dd, $J=12.7,10.6 \mathrm{~Hz}, 1 \mathrm{H}), 2.83$ (dd, $J=16.7,11.1 \mathrm{~Hz}$, $1 \mathrm{H}), 2.97$ (dd, $J=12.9,5.8 \mathrm{~Hz}, 1 \mathrm{H}), 3.30$ (d, $J=13.3 \mathrm{~Hz}$, $1 \mathrm{H}), 3.85$ (s, 3H), 3.89 (s, 3H), 3.97 (t, $J=8.2 \mathrm{~Hz}, 1 \mathrm{H})$, $4.10(\mathrm{~d}, J=8.7 \mathrm{~Hz}, 1 \mathrm{H}), 4.41 \sim 4.47(\mathrm{~m}, 1 \mathrm{H}), 4.52 \sim 4.59$ $(\mathrm{m}, 1 \mathrm{H}), 6.75 \sim 6.77(\mathrm{~m}, 2 \mathrm{H}), 6.87(\mathrm{~s}, 1 \mathrm{H}), 7.26 \sim 7.34(\mathrm{~m}$, $5 \mathrm{H}) ;{ }^{13} \mathrm{C}$ NMR $\left(100 \mathrm{MHz}, \mathrm{CDCl}_{3}\right) \delta: 175.5,171.4,153.2$, $149.0,147.9,135.8,130.7,129.6,129.0,127.4,121.4$, $112.3,111.1,80.9,66.0,56.01,55.97,55.7,41.5,38.0$, 37.6, 36.8, 28.2; ESI-MS m/z: $506.1[\mathrm{M}+\mathrm{Na}]^{+}$.

\section{$3.2 .5(R)$-3-着径基-4-(3,4-二甲氧基苯基)-丁酸叔丁} 酯(7a) 的合成

将上步产物 6a (2.50 g, $5.2 \mathrm{mmol})$ 溶于 $80 \mathrm{~mL}$ 四氢呋 喃中, 加入 $20 \mathrm{~mL}$ 水, 分批加入硽氢化钠 $(0.29 \mathrm{~g}, 5.8$ $\mathrm{mmol}$ ), 室温搅拌 $2 \mathrm{~h}$. 减压浓缩除去大部分四氢呋喃, 乙酸乙酯稀释, 缓慢滴加 $1 \mathrm{~mol} / \mathrm{L}$ 盐酸至无气泡产生. 依次用 $5 \%$ 硫酸氢钾和饱和氯化钠洗涤, 无水硫酸钠干 燥. 抽滤, 滤液减压浓缩, 得 $1.52 \mathrm{~g}$ 无色透明液体 7a. 不经纯化, 可直接用于下步反应. $[\alpha]_{\mathrm{D}}^{25}-10.2$ (c 1.0, $\mathrm{CHCl}_{3}$ ); ${ }^{1} \mathrm{H} \mathrm{NMR}\left(400 \mathrm{MHz}, \mathrm{CDCl}_{3}\right) \delta: 1.45$ (s, 9H), 2.18 (br, $1 \mathrm{H}), 2.23 \sim 2.36(\mathrm{~m}, 3 \mathrm{H}), 2.55(\mathrm{dd}, J=13.6,6.2 \mathrm{~Hz}$, $1 \mathrm{H}), 2.65(\mathrm{dd}, J=13.6,6.2 \mathrm{~Hz}, 1 \mathrm{H}), 3.52(\mathrm{dd}, J=10.4,4.6$ $\mathrm{Hz}, 1 \mathrm{H}), 3.62$ (dd, $J=10.3,3.8 \mathrm{~Hz}, 1 \mathrm{H}), 3.85$ (s, 3H), 3.87 $(\mathrm{s}, 3 \mathrm{H}), 6.66 \sim 6.78(\mathrm{~m}, 2 \mathrm{H}), 6.79(\mathrm{~d}, J=8.6 \mathrm{~Hz}, 1 \mathrm{H}) ;{ }^{13} \mathrm{C}$ NMR $\left(100 \mathrm{MHz}, \mathrm{CDCl}_{3}\right) \delta: 172.4,148.2,146.8,131.6$, 120.6, 111.7, 110.5, 80.1, 64.4, 55.3, 55.2, 39.2, 36.8, 36.3, 27.5; ESI-MS $m / z: 333.2[\mathrm{M}+\mathrm{Na}]^{+}$; HRMS (ESI-TOF) calcd for $\mathrm{C}_{17} \mathrm{H}_{26} \mathrm{O}_{5} \mathrm{Na}[\mathrm{M}+\mathrm{Na}]^{+}$333.1672, found 333.1686 .

3.2 .6 (S)-3-羟甲基-4-(3,4-二甲氧基苯基)-丁酸叔丁 酯(7b) 的合成

同化合物 7a 合成. 无色透明液体. $[\alpha]_{\mathrm{D}}^{25}+9.8(c$ 1.0, $\mathrm{CHCl}_{3}$ ); ${ }^{1} \mathrm{H} \mathrm{NMR}\left(400 \mathrm{MHz}, \mathrm{CDCl}_{3}\right) \delta: 1.45$ (s, 9H), $2.26 \sim 2.34(\mathrm{~m}, 3 \mathrm{H}), 2.55(\mathrm{dd}, J=13.6,6.5 \mathrm{~Hz}, 1 \mathrm{H}), 2.65$ $(\mathrm{dd}, J=13.6,6.6 \mathrm{~Hz}, 1 \mathrm{H}), 3.48 \sim 3.56(\mathrm{~m}, 1 \mathrm{H}), 3.62 \sim 3.73$ $(\mathrm{m}, 1 \mathrm{H}), 3.86(\mathrm{~s}, 3 \mathrm{H}), 3.87(\mathrm{~s}, 3 \mathrm{H}), 6.72 \sim 6.73(\mathrm{~m}, 2 \mathrm{H})$, $6.79(\mathrm{~d}, J=8.6 \mathrm{~Hz}, 1 \mathrm{H}) ;{ }^{13} \mathrm{C} \mathrm{NMR}\left(100 \mathrm{MHz}, \mathrm{CDCl}_{3}\right) \delta$ : 172.3, 148.2, 146.8, 131.6, 120.6, 111.7, 110.5, 80.1, 64.4, 55.3, 55.2, 39.2, 36.8, 36.3, 27.5; ESI-MS m/z: $333.2[\mathrm{M}+$ $\mathrm{Na}]^{+}$.

\subsection{7 (R)-4-(3,4-二甲氧基芐基)-丁内酯 $(\mathbf{8 a})$ 的合成}

将上步产物 7a (1.50 g, $4.8 \mathrm{mmol})$ 溶于 $20 \mathrm{~mL}$ 甲苯, 加入对甲苯磺酸 $(41.6 \mathrm{mg}, 0.24 \mathrm{mmol})$, 于 $80{ }^{\circ} \mathrm{C}$ 搅拌 $1 \mathrm{~h}$. 减压浓缩除去甲苯. 残渣溶于乙酸乙酯, 依次用 $10 \%$ 碳 酸氢钠和饱和氯化钠溶液洗涤, 无水硫酸钠干燥, 硅胶 柱层析 $[V$ (石油醚) $: V($ 乙酸乙酯 $)=2: 1]$, 得 $1.14 \mathrm{~g}$ 无色
透明液体 $8 \mathbf{a}$, 两步产率 $83 \%$, ee 值 $98 \%$ [大赛璐 CHIRALPAK $^{\circledR}$ IF 色谱柱, 以 $V$ (正己烷) : $V$ (异丙醇) : $V($ 叔丁基甲醚 $)=80: 10 ： 10$ 为流动相, 流率 0.8 $\mathrm{mL} / \mathrm{min}$, 检测波长为 UV $220 \mathrm{~nm}$, 温度 $25^{\circ} \mathrm{C} .8 \mathbf{a}$ 的保留 时间为 $47.5 \mathrm{~min}, \mathbf{8 b}$ 的保留时间为 $51.3 \mathrm{~min}]$. [ $\alpha]_{\mathrm{D}}^{25}+6.4$ $\left(c\right.$ 1.0, $\mathrm{CHCl}_{3}$ ) [文献值 ${ }^{[16]}[\alpha]_{\mathrm{D}}+4.3\left(c\right.$ 2.6, $\left.\mathrm{CHCl}_{3}\right)$ ]; ${ }^{1} \mathrm{H}$ NMR (400 MHz, $\left.\mathrm{CDCl}_{3}\right) \delta: 2.30(\mathrm{dd}, J=17.5,6.8 \mathrm{~Hz}$, $1 \mathrm{H}), 2.61$ (dd, $J=17.5,8.1 \mathrm{~Hz}, 1 \mathrm{H}), 2.66 \sim 2.78(\mathrm{~m}, 2 \mathrm{H})$, $2.79 \sim 2.90(\mathrm{~m}, 1 \mathrm{H}), 3.87(\mathrm{~s}, 3 \mathrm{H}), 3.88(\mathrm{~s}, 3 \mathrm{H}), 4.05(\mathrm{dd}$, $J=9.0,6.1 \mathrm{~Hz}, 1 \mathrm{H}), 4.34(\mathrm{dd}, J=9.0,7.1 \mathrm{~Hz}, 1 \mathrm{H}), 6.67$ (s, $1 \mathrm{H}), 6.70(\mathrm{~d}, J=8.1 \mathrm{~Hz}, 1 \mathrm{H}), 6.82(\mathrm{~d}, J=8.1 \mathrm{~Hz}, 1 \mathrm{H}) ;{ }^{13} \mathrm{C}$ NMR $\left(100 \mathrm{MHz}, \mathrm{CDCl}_{3}\right) \delta: 177.0,149.2,148.0,130.9$, 120.8, 111.9, 111.5, 72.7, 56.02, 55.99, 38.7, 37.4, 34.3; ESI-MS $m / z: 237.1[\mathrm{M}+\mathrm{H}]^{+}, 259.1[\mathrm{M}+\mathrm{Na}]^{+}$.

\subsection{8 (S)-4-(3,4-二甲氧基苄基)-丁内酯 $(\mathbf{8 b})$ 的合成}

同化合物 8a 合成. 无色透明液体，两步产率 $81 \%$, $e e$ 值 96\%. [ $\alpha]_{\mathrm{D}}^{25}-6.3$ (c 1.0, $\left.\mathrm{CHCl}_{3}\right) ;{ }^{1} \mathrm{H}$ NMR (400 $\left.\mathrm{MHz}, \mathrm{CDCl}_{3}\right) \delta: 2.29(\mathrm{dd}, J=17.5,6.9 \mathrm{~Hz}, 1 \mathrm{H}), 2.61$ (dd, $J=17.5,8.1 \mathrm{~Hz}, 1 \mathrm{H}), 2.70 \sim 2.77(\mathrm{~m}, 2 \mathrm{H}), 2.80 \sim 2.88(\mathrm{~m}$, $1 \mathrm{H}), 3.86$ (s, 3H), 3.87 (s, 3H), 4.04 (dd, $J=8.9,6.2 \mathrm{~Hz}$, $1 \mathrm{H}), 4.33$ (dd, $J=8.9,7.1 \mathrm{~Hz}, 1 \mathrm{H}), 6.67$ (s, 1H), 6.70 (d, $J=8.2 \mathrm{~Hz}, 1 \mathrm{H}), 6.82(\mathrm{~d}, J=8.0 \mathrm{~Hz}, 1 \mathrm{H}) ;{ }^{13} \mathrm{C}$ NMR $(100$ $\left.\mathrm{MHz}, \mathrm{CDCl}_{3}\right) \delta: 177.0,149.2,148.0,130.9,120.8,111.9$, $111.5,72.8,56.02,56.00,38.7,37.4,34.3$; ESI-MS $\mathrm{m} / \mathrm{z}$ : $237.1[\mathrm{M}+\mathrm{H}]^{+}, 259.1[\mathrm{M}+\mathrm{Na}]^{+}$.

$3.2 .9(3 R, 4 R)$-3-(3-甲氧基-4-芐氧基苄基)-4-(3,4-二 甲氧基苄基) - 丁内酯(10a)的合成

将二异丙胺 $(360.0 \mu \mathrm{L}, 2.5 \mathrm{mmol}$ )溶于 $20 \mathrm{~mL}$ 干燥的 四氢呋喃, 冰浴滴加正丁基锂 $(1.6 \mathrm{~mL}, 2.5 \mathrm{mmol}, 1.6$ $\mathrm{mol} / \mathrm{L}$ in $\mathrm{THF})$, 反应 $30 \mathrm{~min}$ 后降至 $-78{ }^{\circ} \mathrm{C}$, 滴加上步 产物 $8 \mathbf{a}(400.0 \mathrm{mg}, 1.7 \mathrm{mmol})$ 的 $1 \mathrm{~mL}$ 四氢呋喃溶液, 继 续搅拌 $1 \mathrm{~h}$, 滴加化合物 9 (519.0 mg, $1.69 \mathrm{mmol})$ 的 $1 \mathrm{~mL}$ 四氢呋喃溶液, 继续搅拌 $4 \mathrm{~h}$, 饱和氯化铵淬灭反应, 减 压浓缩除去大部分四氢呋喃, 剩余物溶于乙酸乙酯, 依 次用 $5 \%$ 硫酸氢钾和饱和氯化钠洗涤, 无水硫酸钠干燥 后, 硅胶柱层析 $[V$ (石油醚) : $V$ (乙酸乙酯 $)=4: 1]$, 得 $614 \mathrm{mg}$ 无色粘稠液体 10a, 收率 79\%. [ $\alpha]_{\mathrm{D}}^{25}-26.2(c$ 1.3, $\left.\mathrm{CHCl}_{3}\right)\left\{\right.$ 文献值 ${ }^{[17]}[\alpha]_{\mathrm{D}}^{25}-26.8\left(c\right.$ 1.0, $\left.\left.\mathrm{CHCl}_{3}\right)\right\} ;{ }^{1} \mathrm{H}$ NMR $\left(400 \mathrm{MHz}, \mathrm{CDCl}_{3}\right) \delta: 2.45 \sim 2.53(\mathrm{~m}, 2 \mathrm{H}), 2.55 \sim$ $2.62(\mathrm{~m}, 2 \mathrm{H}), 2.87 \sim 2.99(\mathrm{~m}, 2 \mathrm{H}), 3.80(\mathrm{~s}, 3 \mathrm{H}), 3.84(\mathrm{~s}$, $3 \mathrm{H}), 3.85(\mathrm{~s}, 3 \mathrm{H}), 3.88 \sim 3.90(\mathrm{~m}, 1 \mathrm{H}), 4.12(\mathrm{t}, J=7.7 \mathrm{~Hz}$, $1 \mathrm{H}), 5.13(\mathrm{~s}, 2 \mathrm{H}), 6.47(\mathrm{~s}, 1 \mathrm{H}), 6.53(\mathrm{~d}, J=8.0 \mathrm{~Hz}, 1 \mathrm{H})$, $6.59(\mathrm{~d}, J=7.5 \mathrm{~Hz}, 1 \mathrm{H}), 6.71(\mathrm{~s}, 1 \mathrm{H}), 6.74(\mathrm{~d}, J=8.2 \mathrm{~Hz}$, $1 \mathrm{H}), 6.78(\mathrm{~d}, J=7.9 \mathrm{~Hz}, 1 \mathrm{H}), 7.29 \sim 7.43(\mathrm{~m}, 5 \mathrm{H}) ;{ }^{13} \mathrm{C}$ NMR $\left(100 \mathrm{MHz}, \mathrm{CDCl}_{3}\right) \delta: 178.9,149.9,149.2,148.0$, 
$147.2,137.3,131.0,130.6,128.7,128.0,127.8,127.4$, $127.1,121.5,120.7,114.2,113.0,112.0,111.5,71.4,71.2$, $65.5,56.12,56.05,55.96,46.7,41.3,38.3,34.7$; ESI-MS $m / z: 462.9[\mathrm{M}+\mathrm{H}]^{+}, 484.9[\mathrm{M}+\mathrm{Na}]^{+}$.

3.2 .10 (3S,4S)-3-(3-甲氧基-4-芐氧基苄基)-4-(3,4-二甲 氧基苄基) - 丁内酯(10b)的合成

将二异丙胺 $(145 \mu \mathrm{L}, 1.02 \mathrm{mmol})$ 溶于 $15 \mathrm{~mL}$ 干燥的 四氢呋喃, 冰浴滴加正丁基锂 $(0.64 \mathrm{~mL}, 1.02 \mathrm{mmol}, 1.6$ $\mathrm{mol} / \mathrm{L}$ in $\mathrm{THF}$ ), 反应 $30 \mathrm{~min}$ 后降至 $-78{ }^{\circ} \mathrm{C}$, 滴加上步 产物 $8 \mathbf{b}(160.0 \mathrm{mg}, 0.68 \mathrm{mmol})$ 的 $1 \mathrm{~mL}$ 四氢呋喃溶液, 反应 $1 \mathrm{~h}$ 后, 滴加化合物 9 (208.9 mg, $0.68 \mathrm{mmol})$ 的 $1 \mathrm{~mL}$ 四氢呋喃溶液, 继续搅拌 $4 \mathrm{~h}$ 至 TLC 显示反应完全, 饱 和氯化铵淬灭反应, 减压浓缩除去大部分四氢呋喃, 剩 余物溶于乙酸乙酯, 依次用 $5 \%$ 硫酸氢钾和饱和氯化钠 洗涤, 无水硫酸钠干燥后, 硅胶柱层析 $[V$ (石油醚) : $V($ 乙酸乙酯 $)=4: 1]$. 得 $239.0 \mathrm{mg}$ 无色粘稠液体 $\mathbf{1 0 b}$, 收率 76\%, de >99\%. [a $]_{\mathrm{D}}^{25}+26.5$ (c 1.3, $\left.\mathrm{CHCl}_{3}\right) ;{ }^{1} \mathrm{H}$ NMR $\left(400 \mathrm{MHz}, \mathrm{CDCl}_{3}\right) \delta: 2.54 \sim 2.43(\mathrm{~m}, 2 \mathrm{H}), 2.64 \sim$ $2.54(\mathrm{~m}, 2 \mathrm{H}), 2.93(\mathrm{qd}, J=14.1,6.0 \mathrm{~Hz}, 2 \mathrm{H}), 3.80(\mathrm{~s}, 3 \mathrm{H})$, 3.84 (s, 3H), 3.85 (s, 3H), 3.88 (dd, $J=10.9,3.3 \mathrm{~Hz}, 1 \mathrm{H})$, $4.11(\mathrm{dd}, J=9.0,7.0 \mathrm{~Hz}, 1 \mathrm{H}), 5.13(\mathrm{~s}, 2 \mathrm{H}), 6.47$ (d, $J=1.8$ $\mathrm{Hz}, 1 \mathrm{H}), 6.52(\mathrm{dd}, J=8.1,1.8 \mathrm{~Hz}, 1 \mathrm{H}), 6.59$ (dd, $J=8.1$, $1.8 \mathrm{~Hz}, 1 \mathrm{H}), 6.71(\mathrm{~d}, J=1.8 \mathrm{~Hz}, 1 \mathrm{H}), 6.74(\mathrm{~d}, J=8.1 \mathrm{~Hz}$, $1 \mathrm{H}), 6.78(\mathrm{~d}, J=8.1 \mathrm{~Hz}, 1 \mathrm{H}), 7.42 \sim 7.29(\mathrm{~m}, 5 \mathrm{H}) ;{ }^{13} \mathrm{C}$ NMR $\left(100 \mathrm{MHz}, \mathrm{CDCl}_{3}\right) \delta: 178.9,149.9,149.1,148.0$, $147.2,137.3,131.0,130.6,128.7,128.0,127.4,121.5$, $120.7,114.1,113.0,111.9,111.4,71.4,71.2,56.12,56.04$, 55.96, 46.7, 41.3, 38.3, 34.7; ESI-MS $m / z: 462.9[\mathrm{M}+\mathrm{H}]^{+}$, $484.9[\mathrm{M}+\mathrm{Na}]^{+}$.

\section{$3.2 .11(-)$-牛蒡苷元 $(1)$ 的合成}

将上步产物 10a (600.0 mg, $1.3 \mathrm{mmol})$ 溶于 $20 \mathrm{~mL}$ 甲 醇, 加入 $10 \% \mathrm{Pd} / \mathrm{C}(60.0 \mathrm{mg})$, 氢气氛下室温搅拌 $1 \mathrm{~h}$. 抽滤, 滤液减压浓缩得米色固体, 乙醚洗涤数次, 得 $474 \mathrm{mg}$ 白色粉末状固体 1, 产率 98\%,ee 值 97\%[大赛璐 CHIRALPAK $^{\circledR}$ ID 色谱柱, 以 $V$ (正己烷) : $V$ (异丙醇) : $V($ 叔丁基甲醚 $)=80: 10 ： 10$ 为流动相, 流率 0.8 $\mathrm{mL} / \mathrm{min}$, 检测波长为 UV $220 \mathrm{~nm}$, 温度 $25{ }^{\circ} \mathrm{C} .1$ 的保留 时间为 $40.9 \mathrm{~min}, 2$ 的保留时间为 $35.2 \mathrm{~min}$ ]. m.p. 100 $102{ }^{\circ} \mathrm{C} ;[\alpha]_{\mathrm{D}}^{25}-30.7$ (c 1.0, $\mathrm{CHCl}_{3}$ ) [文献值 ${ }^{[18]}$ m.p. $100 \sim 101{ }^{\circ} \mathrm{C},[\alpha]_{\mathrm{D}}^{25}-31.5\left(c 1.06, \mathrm{CHCl}_{3}\right)$, 天然产物]; ${ }^{1} \mathrm{H}$ NMR $\left(400 \mathrm{MHz}, \mathrm{CDCl}_{3}\right) \delta: 2.42 \sim 2.74(\mathrm{~m}, 4 \mathrm{H})$, $2.77 \sim 3.08(\mathrm{~m}, 2 \mathrm{H}), 2.82(\mathrm{~s}, 3 \mathrm{H}), 2.83(\mathrm{~s}, 3 \mathrm{H}), 3.85(\mathrm{~s}$, $3 \mathrm{H}), 3.88(\mathrm{dd}, J=8.9,7.4 \mathrm{~Hz}, 1 \mathrm{H}), 4.14(\mathrm{dd}, J=8.9,7.1$ $\mathrm{Hz}, 1 \mathrm{H}), 5.57(\mathrm{~s}, 1 \mathrm{H}), 6.46(\mathrm{~s}, 1 \mathrm{H}), 6.55(\mathrm{~d}, J=8.0 \mathrm{~Hz}$, $1 \mathrm{H}), 6.59 \sim 6.68(\mathrm{~m}, 2 \mathrm{H}), 6.75(\mathrm{~d}, J=8.1 \mathrm{~Hz}, 1 \mathrm{H}), 6.83(\mathrm{~d}$,
$J=7.9 \mathrm{~Hz}, 1 \mathrm{H}) ;{ }^{13} \mathrm{C} \mathrm{NMR}\left(100 \mathrm{MHz}, \mathrm{CDCl}_{3}\right) \delta: 178.9$, $149.2,148.0,146.8,144.7,130.6,129.6,122.2,120.7$, $114.2,111.9,111.6,111.4,71.5,56.02,55.97,55.93,46.7$, 41.1, 38.3, 34.6; ESI-MS m/z: $373.2[\mathrm{M}+\mathrm{H}]^{+}, 395.1[\mathrm{M}+$ $\mathrm{Na}]^{+}$; HRMS (ESI-TOF) calcd for $\mathrm{C}_{21} \mathrm{H}_{25} \mathrm{O}_{6}[\mathrm{M}+\mathrm{H}]^{+}$ 373.1646 , found 373.1642 .

\section{$3.2 .12(+)$-牛蒡苷元(2)的合成}

同化合物 1 合成. 白色固体，产率 100\%. m.p. 99 $102{ }^{\circ} \mathrm{C}$; ee 值 96\%; $[\alpha]_{\mathrm{D}}^{25}+30.3\left(c 1.0, \mathrm{CHCl}_{3}\right) ;{ }^{1} \mathrm{H} \mathrm{NMR}$ $\left(400 \mathrm{MHz}, \mathrm{CDCl}_{3}\right) \delta: 2.42 \sim 2.74(\mathrm{~m}, 4 \mathrm{H}), 2.77 \sim 3.08(\mathrm{~m}$, $2 \mathrm{H}), 3.82(\mathrm{~s}, 3 \mathrm{H}), 3.83(\mathrm{~s}, 3 \mathrm{H}), 3.85(\mathrm{~s}, 3 \mathrm{H}), 3.88(\mathrm{dd}, J=$ 8.9, $7.4 \mathrm{~Hz}, 1 \mathrm{H}), 4.14(\mathrm{dd}, J=8.9,7.1 \mathrm{~Hz}, 1 \mathrm{H}), 5.57$ (s, $1 \mathrm{H}), 6.46(\mathrm{~s}, 1 \mathrm{H}), 6.55(\mathrm{~d}, J=8.0 \mathrm{~Hz}, 1 \mathrm{H}), 6.59 \sim 6.68(\mathrm{~m}$, $2 \mathrm{H}), 6.75(\mathrm{~d}, J=8.1 \mathrm{~Hz}, 1 \mathrm{H}), 6.83(\mathrm{~d}, J=7.9 \mathrm{~Hz}, 1 \mathrm{H}) ;{ }^{13} \mathrm{C}$ NMR $\left(100 \mathrm{MHz}, \mathrm{CDCl}_{3}\right) \delta: 178.9,149.2,148.0,146.8$, $144.7,130.6,129.6,122.2,120.7,114.2,111.9,111.6$, 111.4, 71.5, 56.02, 55.97, 55.93, 46.7, 41.1, 38.3, 34.6; ESI-MS $m / z: 373.2[\mathrm{M}+\mathrm{H}]^{+}, 395.1[\mathrm{M}+\mathrm{Na}]^{+}$.

\section{2 .13 丁二酸单苄酯(12)的合成}

参照文献[19]所述方法合成，产率 $62 \%$ ，化合物表 征数据与文献一致.

\subsubsection{4 (S)-4-芳基-3-(单芳酯基丁酰基)-2-噁唑烷酮} (13) 的合成

参照 $5 \mathbf{a}$ 的合成方法, 将 3,4-二甲氧基苯丙酸变更为 丁二酸单茮酯, 得无色透明液体, 产率 $97 \%$. $[\alpha]_{\mathrm{D}}^{25}+$ 54.3 (c 1.0, $\left.\mathrm{CHCl}_{3}\right) ;{ }^{1} \mathrm{H}$ NMR (400 MHz, $\left.\mathrm{CDCl}_{3}\right) \delta: 2.82 \sim$ $2.71(\mathrm{~m}, 3 \mathrm{H}), 3.34 \sim 3.21(\mathrm{~m}, 3 \mathrm{H}), 4.21 \sim 4.14(\mathrm{~m}, 2 \mathrm{H})$, $4.67 \sim 4.61(\mathrm{~m}, 1 \mathrm{H}), 5.16(\mathrm{~s}, 2 \mathrm{H}), 7.20 \sim 7.19(\mathrm{~m}, 2 \mathrm{H})$, $7.37 \sim 7.25(\mathrm{~m}, 8 \mathrm{H}) ;{ }^{13} \mathrm{C} \mathrm{NMR}\left(100 \mathrm{MHz}, \mathrm{CDCl}_{3}\right) \delta:$ $172.2,171.8,153.5,135.8,135.2,129.4,129.0,128.6$, 128.2, 127.4, 66.6, 66.3, 55.1, 37.7, 30.8, 28.4; ESI-MS $368.2[\mathrm{M}+\mathrm{H}]^{+}, 390.2[\mathrm{M}+\mathrm{Na}]^{+}$; HRMS (ESI-TOF) calcd for $\mathrm{C}_{21} \mathrm{H}_{21} \mathrm{NO}_{5} \mathrm{Na}[\mathrm{M}+\mathrm{Na}]^{+}$390.1312, found 390.1312 .

致谢 感谢大赛璐药物手性技术(上海)有限公司对化合 物光学纯度的测定.

辅助材料(Supporting Information) 中间体和目标产 物的 ${ }^{1} \mathrm{H} \mathrm{NMR} 、{ }^{13} \mathrm{C} \mathrm{NMR}$ 图谱以及(-)-牛蒡子苷元 1 的 HRMS 图谱. 这些材料可以免费从本刊网站(http://siocjournal.cn/)上下载.

\section{References}

[1] Hyam, S. R.; Lee, I. A.; Gu, W.; Kim, K. A.; Jeong, J. J.; Jang, S. 
E.; Han, M.-J.; Kim, D. H. Eur. J. Pharmacol. 2013, 708, 21.

[2] Zhao, Z.-Y.; Yin, Y.-Q.; Wu, H.; Jiang, M.; Lou, J.-S.; Bai, G.; Luo, G.-A. Cell. Physiol. Biochem. 2013, 32, 1342.

[3] Miele, C.; Beguinot, F. Diabetologia 2012, 55, 1244.

[4] Zhang, N.; Wen, Q.-P.; Ren, L.; Liang, W.-B.; Xia, Y.; Zhang, X.-D; Zhao, D.; Sun, D.; Hu, Y.; Hao, H.-G.; Yan, Y.-P.; Zhang, G.-X.; Yang, J.-X.; Kang, T.-G. Int. J. Mol. Sci. 2013, 14, 18657.

[5] Gu, Y.; Scheuer, C.; Feng, D.-L; Menger, M. D.; Laschke, M. W. Anti-Cancer Drug 2013, 8, 781.

[6] Gu, Y.; Qi, C.-T.; Sun, X.-X.; Ma, X.-Q.; Zhang, H.-H.; Hu, L.-H.; Yuan, J.-Y.; Yu, Q. Acta Pharmacol. Sin. 2012, 33, 941.

[7] Zhu, Z.-Y.; Yan, J.-M.; Jiang, W.; Yao, X.-G.; Chen, J.; Chen, L.-L.; Li, C.-J.; Hu, L.-H.; Jiang, H.-L.; Shen, X. J. Neurosci. 2013, 33, 13138.

[8] Landete, J. M. Food Res. Int. 2012, 46, 410.

[9] (a) Wang, H.-H.; Wu, P.; Kang, H.; Xu, L.; Zhu, R.-X.; Kang, T.-G. Chin. J. Org. Chem. 2012, 32, 1894 (in Chinese). (王欢欢, 吴平, 康宏, 许亮, 朱瑞新, 康廷国, 有机化学, 2012, 32, 1894.)

(b) Xu, Y.-B.; Dou, D.-Q. J. Liaoning Univ. Tradit. Chin. Med. 2013, (7), 64 (in Chinese).

(徐显涁, 窦德强, 辽宁中医药大学学报, 2013, (7), 64.)

(c) Chen, G.-R.; Li, H.-F.; Dou, D.-Q; Xu, Y.-B.; Jiang, H.-S.; Li, F.-R.; Kang, T.-G. Nat. Prod. Res. 2013, 23, 2251.

[10] Li, D.-W.; Liu, Q.-P.; Jia, D.; Dou, D.-Q.; Wang, X.-F.; Kang, T.-G. Planta Med. 2014, 80, 48.

[11] (a) Amancha, P. K.; Liu, H.-J.; Ly, T. W.; Shia, K. S. Eur. J. Org. Chem. 2010, 18, 3473.

(b) Eich, E.; Pertz, H.; Kaloga, M.; Schulz, J.; Fesen, M. R.; Ma- zumder, A.; Pommier, Y. J. Med. Chem. 1996, 39, 86.

(c) Bode, J. W.; Doyle, M. P.; Protopopova, M. N.; Zhou, Q.-L. J. Org. Chem. 1996, 61, 9146.

(d) Fischer, J.; Reynolds, A. J.; Sharp, L. A.; Sherburn, M. S. Org. Lett. 2004, 6, 1345.

(e) Pohmakotr, M.; Soorukram, D.; Tuchinda, P.; Prabpai, S.; Kongsaeree, P.; Reutrakul, V. Tetrahedron Lett. 2004, 45, 4315.

[12] Lin, G.-Q.; Chen, Y.-Q.; Chen, X.-Z.; Lin, Y.-M. Chiral Synthesis, Asymmetric Reaction and Its Application, Beijing Science Press, Beijing, 2000, p. 56 (in Chinese).

(林国强, 陈耀全，陈新滋，李月明，手性合成——不对称反应 及其应用, 科学出版社, 北京, 2000, p. 56.)

[13] Markus, N.; Christine, W.; Tobias, B.; Felix, M.; Jens, C.; Benedikt, S.; Ralf, P.; Soledad, R. G.; Thomas, P.; Norbert, S. J. Med. Chem. 2013, 56, 1853.

[14] Woo, L. W. L.; Bubert, C.; Sutcliffe, O. B.; Smith, A.; Chander, S. K.; Mahon, M. F.; Purohit, A.; Reed, M. J.; Potter, B. V. L. J. Med. Chem. 2007, 50, 3540.

[15] So, M.; Kotake, T.; Matsuura, K.; Inui, M.; Kamimura, A. J. Org. Chem. 2012, 77, 4017.

[16] Hughes, G.; Kimura, M.; Buchwald, S. L. J. Am. Chem. Soc. 2003, $125,11253$.

[17] Sibi, M. P.; Liu, P.; Ji, J.; Hajra, S.; Chen, J. J.Org. Chem. 2002, 67, 1738

[18] Moritani, S.; Nomura, M.; Takeda, Y.; Miyamoto, K. Biol. Pharm. Bull. 1996, 19, 1515.

[19] Chen, X.-Y.; Zenger, K.; Lupp, A.; Kling, B.; Heilmann, J.; Fleck, C.; Kraus, B.; Decker, M. J. Med. Chem. 2012, 55, 5231.

(Li, L.; Lu, Z.) 